\title{
How to renormalize integral equations with singular potentials in effective field theory
}

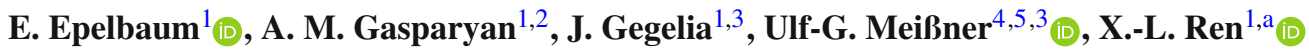 \\ ${ }^{1}$ Faculty of Physics and Astronomy, Institute for Theoretical Physics II, Ruhr University Bochum, 44870 Bochum, Germany \\ ${ }^{2}$ NRC "Kurchatov Institute", ITEP, B. Cheremushkinskaya 25, 117218 Moscow, Russia \\ 3 Tbilisi State University, 0186 Tbilisi, Georgia \\ ${ }^{4}$ Helmholtz Institut für Strahlen- und Kernphysik and Bethe Center for Theoretical Physics, Universität Bonn, 53115 Bonn, Germany \\ ${ }^{5}$ Institute for Advanced Simulation, Institut für Kernphysik and Jülich Center for Hadron Physics, Forschungszentrum Jülich, 52425 Jülich, \\ Germany
}

Received: 24 January 2020 / Accepted: 5 May 2020 / Published online: 26 May 2020

(C) The Author(s) 2020

Communicated by Vittorio Somà

\begin{abstract}
We briefly review general concepts of renormalization in quantum field theory and discuss their application to solutions of integral equations with singular potentials in the few-nucleon sector of the low-energy effective field theory of QCD. We also describe a particular subtractive renormalization scheme and consider a specific application to a toy-model with a singular potential serving as its effective field theoretical leading-order approximation.
\end{abstract}

\section{Introduction}

The issue of renormalization is a subject of long-standing controversy in the few-nucleon sector of the low-energy effective field theory (EFT) of the strong interactions, pioneered in Ref. [1]. Recent reviews and references addressing this problem can be found, e.g., in Refs. [2-10]. The Lagrangian of any consistent EFT includes all local interactions allowed by the underlying symmetries. Therefore, all ultraviolet (UV) divergences appearing in physical quantities are in principle canceled by the corresponding counterterms [11]. However, the practical implementation of such a quantum field theoretical renormalization is a highly non-trivial problem in the few-body sector of the low-energy EFT of the strong interactions. The difficulty is caused by the fact that due to the large absolute values of the scattering lengths, an infinite number of renormalized diagrams has to be summed up at any order. To perform this summation one defines an effective potential as a sum of irreducible diagrams contributing to the process under consideration and then obtains the scattering amplitude by solving the corresponding integral

a e-mail: xiulei.ren@rub.de (corresponding author) equation [1]. In practice, solving integral equations corresponds to summing up regularized but not renormalized diagrams. Regularized non-perturbative expressions obtained by solving such integral equations, when expanded in powers of $\hbar$ (corresponding to the loop expansion), reproduce the regularized perturbative diagrams. While non-perturbative solutions to integral equations may indeed contain pieces which yield vanishing contributions to the perturbative series, the integral equations in the low-energy EFT are used solely as tools for performing partial resummations of diagrams. Therefore, properly renormalized non-perturbative expressions in a self-consistent EFT must reproduce the renormalized perturbative series, when expanded in $\hbar$.

It is well known how to renormalize Feynman diagrams in quantum field theories. However, it turns out to be a chalenging problem to properly renormalize the non-perturbative solutions to integral equations since this usually requires taking into account contributions of an infinite number of counter terms. The problem is actually caused by the nonrenormalizability of EFT in the traditional sense, manifested by the presence of singular potentials in the few-body sector.

The low-energy EFT of the strong interactions yields a systematic expansion of the effective potentials in terms of small masses and momenta, where small is taken relative to the typical hard (breakdown) scale of about $500 \mathrm{MeV}$. However, these expressions are to be trusted only at low energies. Translated into coordinate space, the EFT yields an expansion of the potential applicable at large distances, while the (strongly scheme-dependent) short-range part is determined by the desired resolution. Accordingly, extrapolations of the long-range part of the effective potential to short distances do not root in the underlying QCD. Moreover, QCD features only shallow bound states in multi-nucleon systems thus indi- 
cating that the singular van der Waals-like behavior of oneand multi-pion exchange potentials is not a valid approximation of the nuclear interaction at short distances.

Reading the literature in the few-body sector of lowenergy chiral EFT and references therein, one often gets the impression that there is no well-established concept of renormalization for non-perturbative problems in quantum field theories, and that we are currently seeking for such a self-consistent definition. In this quest for the solution of a technically challenging problem, fundamental concepts that are well established and should be well-known to the practitioners are often ignored or even disputed in the nuclear physics community. Our understanding is that the very definition of the EFT and renormalization as it is understood in quantum field theory (QFT) specifies uniquely what needs to be done. The implementation of a proper quantum field theoretical renormalization is, however, a rather challenging problem and subject to further investigations.

In our recent work on the renormalization in the few-body sector of the EFT of the strong interactions [12] we have put the main emphasis on discussing details of common misconceptions, i.e. what should not be done when renormalizing the solutions to integral equations with singular EFT potentials. To summarize, the origin of the differences can be traced back to the application or enforcement of quantum mechanical renormalization to a quantum field theoretical problem. This (in our opinion, incorrect) point of view is described in detail in a recent review article [14], see also references therein. It amounts to handling singular potentials emerging in EFT contexts as if they were quantum mechanical potentials valid at all distances rather than large-distance approximations to infinite series. We refer the reader to Ref. [12] for more details.

In this paper we again revisit the issue of renormalization in few-body sector of chiral EFT, but focus on what should be done to achieve the proper renormalization in EFT with singular potentials.

Our paper is organized as follows. In Sect. 2 we discuss the definition and conceptual issues of renormalization in QFTs. In Sect. 3 we consider a particular QFT renormalization scheme which can be applied to the solutions of integral equations with singular potentials. An application of the introduced renormalization scheme to a singular potential for a specific toy model is given in Sect. 4. We summarize our work in Sect. 5.

\section{Renormalization}

Renormalization is a very natural and familiar procedure which is actually carried out in most theories. Considering a theory depending on the parameters $g_{i}$, with $i=1, \ldots, N$, one fixes these parameters from $N$ experiments and makes predictions for the results of other experiments. The standard example is QED, where the electron charge and mass need to be determined from experiment and then e.g. the anomalous magnetic moment of the electron can be predicted to high precision. In mathematical language this means that we calculate $N$ "reference" physical quantities $\sigma_{i}(E)=f_{i}\left(\hbar, g_{1}, g_{2}, \ldots, g_{N}, E\right)$, for $i=1, \ldots, N$, as functions of the couplings $g_{k}$, of kinematical variables collectively denoted by $E$, and $\hbar$, taken to be zero for any classical theory. By taking the expressions of these physical quantities for some fixed kinematics $\mu_{j}(j=1, \ldots, N)$

$$
\begin{aligned}
\sigma_{1}\left(\mu_{1}\right)= & f_{1}\left(\hbar, g_{1}, g_{2}, \ldots, g_{N}, \mu_{1}\right), \\
\sigma_{2}\left(\mu_{2}\right)= & f_{2}\left(\hbar, g_{1}, g_{2}, \ldots, g_{N}, \mu_{2}\right), \\
& \ldots \\
\sigma_{N}\left(\mu_{N}\right)= & f_{N}\left(\hbar, g_{1}, g_{2}, \ldots, g_{N}, \mu_{N}\right),
\end{aligned}
$$

we express the $g_{i}$ as functions of observables $\sigma_{i}\left(\mu_{i}\right)$

$$
\begin{gathered}
g_{i}=\phi_{i}\left(\hbar, \sigma_{1}\left(\mu_{1}\right), \sigma_{2}\left(\mu_{2}\right), \ldots, \sigma_{N}\left(\mu_{N}\right),\right. \\
\left.\mu_{1}, \ldots, \mu_{N}\right), i=1, \ldots, N
\end{gathered}
$$

Next, we substitute the $g_{i}$ from Eq. (1) in the calculated expressions of all physical quantities and obtain

$$
\begin{array}{r}
\sigma_{1}(E)=F_{1}\left(\hbar, \sigma_{1}\left(\mu_{1}\right), \sigma_{2}\left(\mu_{2}\right), \ldots,\right. \\
\left.\sigma_{N}\left(\mu_{N}\right), \mu_{1}, \ldots, \mu_{N}, E\right), \\
\sigma_{2}(E)=F_{2}\left(\hbar, \sigma_{1}\left(\mu_{1}\right), \sigma_{2}\left(\mu_{2}\right), \ldots,\right. \\
\left.\sigma_{N}\left(\mu_{N}\right), \mu_{1}, \ldots, \mu_{N}, E\right), \\
\ldots \\
\sigma_{N}(E)=F_{N}\left(\hbar, \sigma_{1}\left(\mu_{1}\right), \sigma_{2}\left(\mu_{2}\right), \ldots,\right. \\
\sigma_{N+1}(E)=F_{N+1}\left(\hbar, \sigma_{1}\left(\mu_{1}\right), \sigma_{2}\left(\mu_{2}\right), \ldots,\right. \\
\cdots,
\end{array}
$$

where the $F_{i}$ are some functions, whose specific form depends on the considered theory. In this way we express all physical quantities in terms of the "reference" physical quantities taken at the normalization points $\mu_{i}$, instead of the original "bare" parameters. Performed in the framework of QFT this procedure is known as renormalization. Notice that renormalization is non-perturbative if calculations are done non-perturbatively and perturbative if we are using perturbation theory.

As a rule in QFTs, the coefficients of the expansion of physical quantities in powers of the bare couplings $g_{i}$ are divergent. In some theories divergences disappear after renormalization, that is, the expansion of all physical quantities in terms of the "reference" physical quantities in the limit of a removed regulator turn out to have finite coefficients to all orders. Such theories are called renormalizable (as e.g. the 
already mentioned case of QED). To render the Green functions finite one often introduces renormalized fields and corresponding field renormalization constants. However, physical quantities do not depend on the choice of fields and, therefore, one can also work with bare fields. To simplify the discussion we formulate our considerations in such a framework.

In practice one uses more conveniently chosen functions $g_{i}^{R}(\mu)=\mathcal{G}_{i}\left(\sigma_{1}\left(\mu_{1}\right), \sigma_{2}\left(\mu_{2}\right), \ldots, \sigma_{N}\left(\mu_{N}\right), \mu_{1}, \ldots, \mu_{N}\right)$ $(j=1, \ldots, N)$ as new renormalized parameters, where $\mu$ stands collectively for all $\mu_{1}, \ldots, \mu_{N}$. Expressing the original parameters $g_{i}$ in terms of the $g_{i}^{R}(\mu)$

$$
\begin{aligned}
g_{i}=\phi_{i}\left(\hbar, g_{1}^{R}(\mu), g_{2}^{R}(\mu), \ldots,\right. & \\
& \left.g_{N}^{R}(\mu), \mu_{1}, \ldots, \mu_{N}\right), i=1, \ldots, N,
\end{aligned}
$$

and substituting in the Lagrangian of the given QFT, we can express the Lagrangian in terms of the renormalized parameters. Usually, the functions $\mathcal{G}_{i}$ are chosen such tha

$\phi_{i}\left(0, g_{1}^{R}(\mu), g_{2}^{R}(\mu), \ldots, g_{N}^{R}(\mu), \mu_{1}, \ldots, \mu_{N}\right)=g_{i}^{R}(\mu)$,

leading to

$$
\begin{aligned}
g_{i}= & g_{i}^{R}(\mu)+\left[\phi _ { i } \left(\hbar, g_{1}^{R}(\mu), g_{2}^{R}(\mu), \ldots,\right.\right. \\
& \left.g_{N}^{R}(\mu), \mu_{1}, \ldots, \mu_{N}\right)-\phi_{i}\left(0, g_{1}^{R}(\mu), g_{2}^{R}(\mu), \ldots,\right. \\
& \left.\left.g_{N}^{R}(\mu), \mu_{1}, \ldots, \mu_{N}\right)\right] \\
= & g_{i}^{R}(\mu)+\sum_{k=1}^{\infty} \hbar^{k} \delta g_{i}^{k}\left(g_{1}^{R}(\mu), g_{2}^{R}(\mu), \ldots,\right. \\
& \left.g_{N}^{R}(\mu), \mu_{1}, \ldots, \mu_{N}\right),
\end{aligned}
$$

where the $\delta g_{i}^{k}$ are counterterms of $k$-th order in the loop expansion which themselves can be expanded in powers of renormalized coupling constants. Using the Lagrangian parameterized in terms of renormalized couplings and counterterms, we perform calculations of physical quantities in terms of the renormalized parameters $g_{i}^{R}(\mu)$. In renormalizable theories, a perturbative calculation of physical quantities to any finite order in the renormalized coupling constants leads to Taylor series with finite coefficients. All counterterm contributions up to given order in the loop expansion and in the coupling constants have to be taken into account in these calculations. If, for whatever reason, the expansion in any of these renormalized couplings needs to be summed up to an infinite order, then this has to be done systematically by also including the contributions stemming from all counterterms. While such a calculation might be technically very complicated or even unfeasible, the formalism of QFT renormalization defines very precisely and uniquely what has to be done.

From the modern point of view, renormalizable (in the traditional sense) theories are viewed as leading order approximations to effective field theories. The corresponding Lagrangians contain an infinite number of terms compatible with underlying symmetries. A self-consistent EFT is renormalizable in the sense that all divergences appearing in physical quantities can be absorbed in the redefinition of an infinite number of parameters of the effective Lagrangian (i.e. $N$ is equal to infinity in an EFT). Despite the dependence on an infinite number of parameters, EFTs do not loose predictive power if it is possible to find renormalization schemes that ensure that low-energy observables calculated with a specified accuracy depend only on a finite number of renormalized parameters. Notice that physical quantities calculated to some finite order depend on the choice of the renormalization scheme. While this dependence is formally of higher order in the EFT expansion, the higher order corrections to physical quantities are suppressed only for appropriately chosen renormalization conditions. While it is always possible to choose a scheme that destroys the validity of perturbation theory, it is not always possible to find a scheme for which perturbative series make sense. A well-known example is QCD, where, on the one hand, perturbation theory becomes very useful at high energies, due to the asymptotic vanishing of the renormalized coupling, provided the renormalization scale is chosen of the order of the characteristic energy. On the other hand, it is impossible to find a renormalization scheme at low energies which would lead to meaningful perturbative expressions for physical quantities in powers of the renormalized coupling constant.

To be more specific, the Lagrangian of an EFT has the generic form

$\mathcal{L}=K(\psi)+\sum_{i=1}^{\infty} g_{i} O_{i}(\psi)$,

where $K$ is the kinetic part, $O_{i}$ are interaction terms with bare couplings $g_{i}$ and $\psi$ stands collectively for the fields. ${ }^{1}$ Because of the UV divergences one needs to introduce some kind of regularization. The final expressions of physical quantities in terms of the renormalized couplings are finite in the limit of removed regularization and the results do not depend on the choice of the specific regularization scheme. As mentioned above, to render the Green functions finite one often introduces renormalized fields and corresponding field renormalization constants, however, physical quantities do not depend on the choice of fields and therefore one can also work with bare ones in the regularized theory. To calculate physical quantities one expresses the bare couplings in terms of the renormalized ones as specified above and rewrites the Lagrangian as

\footnotetext{
${ }^{1}$ It is understood that the considered EFT leads to a systematic perturbative expansion of physical quantities for energies $E \ll Q$, where $Q$ is some large scale.
} 


$$
\begin{gathered}
\mathcal{L}=K(\psi)+\sum_{i=1}^{\infty}\left[g_{i}^{R}(\mu)+\sum_{k=1}^{\infty} \hbar^{k} \delta g_{i}^{k}\left(g_{1}^{R}(\mu), g_{2}^{R}(\mu), \ldots,\right.\right. \\
\left.\left.g_{N}^{R}(\mu), \mu_{1}, \ldots, \mu_{N}, \Lambda\right)\right] O_{i}(\psi),
\end{gathered}
$$

where we also indicate the dependence of the counterterms on the regularization scheme by including the parameter $\Lambda$ in the list of arguments. While one obviously has a huge freedom of choosing the renormalization conditions, performing consistent calculations of physical quantities up to any order (either finite or infinite) in any coupling $g_{j}^{R}$ for any particular choice requires the inclusion of all contributions up to a given order, generated by the Lagrangian of Eq. (5). It is difficult to implement such a formally consistent program in the few-body sector of the low-energy EFT of the strong interactions, where one needs to sum up an infinite number of diagrams. The problem is caused by the necessity of taking into account contributions of an infinite number of counterterms with growing complexity of the operator structures $O_{i}(\psi)$ in the (heavy baryon) formulation of the EFT.

The best available approach to the non-relativistic formulation of an EFT with pions and nucleons as explicit degrees of freedom is the cutoff EFT which relies on the inclusion of a finite number of counterterms and keeping the cutoff parameter such that the difference between the properly renormalized and actually obtained results is of a higher order than the accuracy of the current calculation [4,13,16-20].

A convenient way of implementing the quantum field theoretical renormalization is to apply the BPHZ subtractive procedure using Zimmerman's forest formula, see, e.g., Ref. [21]. This subtractive approach is equivalent to the above outlined renormalization by including the contributions of all counterterms appearing at a given order. Within the subtractive approach, instead of explicitly including the counterterm contributions, one renormalizes the loop diagrams by subtracting sub-divergences and overall divergences using some fixed renormalization conditions and treats all coupling constants as renormalized finite parameters. In the next section we consider a particular subtractive quantum field theoretical renormalization for the non-perturbative solutions to integral equations with singular effective potentials which removes all divergences from all diagrams generated by iterations of the integral equations.

\section{Integral equation with a singular potential and renormalization}

In this section we consider one particular realization of a subtractive EFT renormalization for solutions of integral equations with singular potentials. Our scheme is a generalization of the one employed in Refs. $[1,22]$ to the case of the onepion-exchange potential and differs from those of Refs. [2327]. In particular, Refs. [26,27] apply a subtraction scheme at vanishing energy, whose realization in terms of local counterterms of the effective Lagrangian is unclear to us. On the other hand Refs. [23-25] demand that the scattering amplitude calculated at a given order must be independent of the subtraction point. As emphasized in the previous section, our understanding is that perturbative amplitudes calculated up to any finite order are renormalization-scheme dependent. A toy-model example considered in the next section demonstrates how problematic it may turn out to find a renormalization scheme, which is not only conceptually consistent but also useful in describing the data.

We emphasize that there is a conceptual difference between the EFT renormalization of the scattering amplitude obtained by solving the Lippmann-Schwinger (LS) equation with singular potentials, that takes into account contributions of an infinite number of counterterms with operator structures of increasing complexity in each partial wave, and selfadjoint extensions of singular Hamiltonians in the framework of quantum mechanics, that ensure finiteness of the calculated observables in the removed regularization limit [28].

We start by considering the LS integral equation for an off-shell scattering amplitude which we write symbolically as

$T=V+\hbar V G T$

To avoid any non-analytic dependence on the quark masses of the counterterms in the few-body sector of the chiral EFT, we split the potential into a singular $V_{S}$ and a regular $V_{R}$ part,

$V=V_{S}+V_{R}$,

where $V_{S}$ has only a polynomial dependence on the quark masses and $V_{R}$ is such that its overlap in loop diagrams with $V_{S}$, i.e. $V_{S} G V_{R}$, is finite. For the potential of Eq. (7) the solution to Eq. (6) can be written in the form

$T=T_{S}+\left(1+\hbar T_{S} G\right) T_{C}\left(1+\hbar G T_{S}\right)$,

where $T_{S}$ and $T_{C}$ satisfy the equations

$T_{S}=V_{S}+\hbar V_{S} G T_{S}$,

and

$T_{C}=V_{R}+\hbar V_{R} G\left(1+\hbar T_{S} G\right) T_{C}$.

To carry out subtractive renormalization, we take into account contributions of counter terms such that the amplitude $T_{S}$ in Eq. (8) is replaced by the renormalized amplitude $T_{S}^{r}$. In particular, we consider iterations of Eq. (9) and perform BPHZ subtractions in each of an infinite number of terms such that the renormalized series has the form: 


$$
\begin{aligned}
T_{S}^{r}= & V_{S}+\hbar V_{S}\left(G-G_{e}\right) V_{S}+\hbar^{2} V_{S}\left(G-G_{e}\right) \\
& \times V_{S}\left(G-G_{e}\right) V_{S}+\cdots .
\end{aligned}
$$

In the first iteration, $\hbar V_{S}\left(G-G_{e}\right) V_{S}$, the result of the original one-loop diagram $\hbar V_{S} G V_{S}$ is expanded around $E=-E_{\mu}$ and the first $N+1$ terms are subtracted by adding $\hbar V_{S}\left(-G_{e}\right) V_{S}$, where

$G_{e}=\left.\sum_{i=0}^{N} \frac{1}{i !}\left(E+E_{\mu}\right)^{i} \frac{d^{i} G(E)}{(d E)^{i}}\right|_{E=-E_{\mu}}$.

That is, we expand $G \equiv G(E)$ at a fixed value $E=-E_{\mu}$ and subtract first several terms, the number of the subtracted terms depending on the UV behaviour of the singular potential. The $N+1$ terms, subtracted at one-loop order, if expanded in powers of momenta and energy, correspond to an infinite number of local counterterms in the effective Lagrangian. Notice here that from the point of view of UV divergences, it would be sufficient to subtract only first several terms in the expansion in powers of momenta and energy. Thus our choice of the renormalization scheme corresponds to finite over-subtractions. This applies to all orders in the loop expansion. In the second iteration in Eq. (11), two one-loop sub-divergences are subtracted from the original two-loop diagram $\hbar^{2} V_{S} G V_{S} G V_{S}$ by adding $\hbar^{2} V_{S}\left(-G_{e}\right) V_{S} G V_{S}$ and $\hbar^{2} V_{S} G V_{S}\left(-G_{e}\right) V_{S}$, and then the overall divergence is subtracted by adding $\hbar^{2} V_{S} G_{e} V_{S} G_{e} V_{S}$. Again, these overall two-loop subtractions correspond to taking into account contributions of an infinite number of counter-terms. Renormalization works analogously for further iterations. The renormalized amplitude $T_{S}^{r}$ satisfies the equation

$T_{S}^{r}=V_{S}+\hbar V_{S}\left(G-G_{e}\right) T_{S}^{r}$.

The reason why we over-subtract each term in series of Eq. (11) is that only this way we can obtain the full renormalized amplitude in closed form as a solution to an integrals equation.

The effective potential $V_{e}$ which includes all necessary counterterms subtracting sub-divergences and overall divergences in all iterations of Eq. (13) can be obtained by solving the following equation

$V_{e}=V_{S}-\hbar V_{S} G_{e} V_{e}$.

That is, the subtracted amplitude $T_{S}^{r}$ can also be obtained by solving the equation

$T_{S}^{r}=V_{e}+\hbar V_{e} G T_{S}^{r}$,

where $V_{e}$ is obtained by solving Eq. (14).

The final renormalized expression of the amplitude has the form

$T^{r}=T_{S}^{r}+\left(1+\hbar T_{S}^{r} G\right) T_{C}^{r}\left(1+\hbar G T_{S}^{r}\right)$, where $T_{C}^{r}$ satisfies the equation

$T_{C}^{r}=V_{R}+\hbar V_{R} G\left(1+\hbar T_{S}^{r} G\right) T_{C}^{r}$.

For practical applications it is convenient to obtain the final renormalized amplitude $T^{r}$ in a single step as a solution to an integral equation. Below we show that indeed $T^{r}$ can be obtained by solving the following integral equation

$T^{r}=V_{S}+\tilde{V}_{R}+\hbar\left(\tilde{V}_{S}+\tilde{V}_{R}\right) G T^{r}$,

where $\tilde{V}_{S}=V_{S}\left(G-G_{e}\right) / G$ and $\tilde{V}_{R}=V_{R}+\hbar\left(V_{S}-\right.$ $\left.\tilde{V}_{S}\right) G V_{R} \cdot{ }^{2}$ To this end we write the solution to Eq. (18) as $T_{S}^{r}+T_{1}$ and show that $T_{1}$ is identical to the second term in the right hand side of Eq. (16). Indeed, substituting $T_{S}^{r}+T_{1}$ in Eq. (18) we obtain the following equation for $T_{1}$

$T_{1}=\tilde{V}_{R}\left(1+\hbar G T_{S}^{r}\right)+\hbar\left(\tilde{V}_{S}+\tilde{V}_{R}\right) G T_{1}$.

Next, we define the amplitude $T_{x}$ via

$T_{1}=:\left(1+\hbar T_{S}^{r} G\right) T_{x}\left(1+\hbar G T_{S}^{r}\right)$

and obtain from Eq. (19)

$$
\begin{gathered}
\left(1-\hbar \tilde{V}_{S} G+\hbar\left(1-\hbar \tilde{V}_{S} G\right) T_{S}^{r} G\right) T_{x} \\
=\tilde{V}_{R}+\hbar \tilde{V}_{R} G\left(1+\hbar T_{S}^{r} G\right) T_{x} .
\end{gathered}
$$

Writing the equation (13) in the form

$\left(1-\hbar \tilde{V}_{S} G\right) T_{S}=V_{S}$,

and using it in Eq. (21), we obtain

$$
\begin{aligned}
\left(1-\hbar \tilde{V}_{S} G+\hbar V_{S} G\right) T_{x}= & \tilde{V}_{R}+\hbar \tilde{V}_{R} G \\
& \times\left(1+\hbar T_{S}^{r} G\right) T_{x} .
\end{aligned}
$$

Using the definition of $\tilde{V}_{R}$, Eq. (23) reduces to

$T_{x}=V_{R}+\hbar V_{R} G\left(1+\hbar T_{S}^{r} G\right) T_{x}$.

Comparing Eqs. (24) and (17), we see that $T_{x}$ is identical with $T_{C}^{r}$ and hence, as evidenced from Eq. (20), $T_{1}$ is identical to the second term in the right hand side of Eq. (16). This proves that indeed $T^{r}$ satisfies Eq. (18).

We emphasize that we consider the above subtractive scheme not because it is amongst the best ones for, e.g., nucleon-nucleon scattering in chiral EFT, but rather since it can be easily applied in practice and, therefore, well suited for demonstration purposes. As noticed in Refs. [29,30] in the nucleon-nucleon scattering problem, it is preferable to use subtraction schemes with two scales corresponding to the leading- and higher-order potentials.

\footnotetext{
${ }^{2}$ Eq. (18) is convenient because it contains only convergent integrals.
} 


\section{Application to a toy model}

As a specific toy-model example of how to apply the discussed renormalization scheme, we construct an effective potential for the underlying "fundamental" potential considered in Ref. [12]

$$
\begin{aligned}
V(r)= & \frac{\alpha\left(e^{-m_{1} r}-e^{-M r}\right)}{r^{3}} \\
& +\frac{\alpha\left(m_{1}-M\right) e^{-m_{1} r}}{r^{2}} \\
& +\frac{\alpha\left(M-m_{1}\right)^{2} e^{-m_{2} r}}{2 r} \\
& -\frac{1}{6} \alpha\left(2 m_{1}-3 m_{2}+M\right)\left(M-m_{1}\right)^{2} e^{-m_{1} r},
\end{aligned}
$$

where $M$ is the light mass and the heavy masses $m_{1}, m_{2}$ represent the large scales. Our choice of parameters is $\alpha=$ $-36 \mathrm{GeV}^{-2}, M=0.1385 \mathrm{GeV}, m_{1}=0.75 \mathrm{GeV}$ and $m_{2}=$ $1.15 \mathrm{GeV}$. The strength of the interaction $\alpha$ is taken equal for all terms, so that the potential $V(r)$ vanishes for $r \rightarrow 0$ and it behaves as $-\alpha e^{-M r} / r^{3}$ for large $r$. More details on this model can be found in Ref. [12].

We consider the LS equation for the S-wave scattering amplitude in the center-of-mass frame of two particles with unit masses

$$
\begin{aligned}
t_{E}\left(p, p^{\prime}\right)= & v\left(p, p^{\prime}\right)+\int_{0}^{\infty} \frac{d q q^{2}}{2 \pi^{2}} v(p, q) \\
& \times \frac{1}{E-q^{2}+i \epsilon} t_{E}\left(q, p^{\prime}\right),
\end{aligned}
$$

with $E=k^{2} / 1 \mathrm{GeV}$ being the total energy, and $p=|\mathbf{p}|$, $p^{\prime}=\left|\mathbf{p}^{\prime}\right|$ the relative momenta of the incoming and outgoing particles, respectively. We set $\hbar=1$ in these calculations.

It is easy to construct the corresponding EFT for the underlying "fundamental" potential in Eq. (25). The LO EFT interaction consists of a delta potential and the long-range part $-\alpha e^{-M r} / r^{3}$, which is singular if extended to the small- $r$ region. Following Ref. [12], we choose the coupling constant $\alpha=-36 \mathrm{GeV}^{-2} \approx-1 /(0.167 \mathrm{GeV})^{2}$ such that the full $\mathrm{LO}$ potential is non-perturbative for the momenta $k \sim M=0.1385 \mathrm{GeV}$. A simple UV analysis shows that the LO potential is perturbatively non-renormalizable, i.e. to remove the divergences from its iterations one needs to introduce counterterms of higher orders (in momenta and energy) which themselves generate new divergences, etc., up to infinity.

We apply the renormalization scheme described in the previous section to the $\mathrm{LO}$ effective potential, for which we take

$$
\begin{aligned}
V_{S}(r)= & c \delta^{(3)}(\mathbf{r})-\frac{\alpha e^{-\mu_{1} r}}{r^{3}}+\frac{\alpha\left(M-\mu_{1}\right) e^{-\mu_{1} r}}{r^{2}} \\
& -\frac{\alpha\left(M-\mu_{1}\right)^{2} e^{-\mu_{1} r}}{2 r},
\end{aligned}
$$

$$
\begin{aligned}
V_{R}(r)= & -\frac{\alpha\left(e^{-M r}-e^{-\mu_{1} r}\right)}{r^{3}}-\frac{\alpha\left(M-\mu_{1}\right) e^{-\mu_{1} r}}{r^{2}} \\
& +\frac{\alpha\left(M-\mu_{1}\right)^{2} e^{-\mu_{1} r}}{2 r} \\
= & \frac{1}{6} \alpha\left(M-\mu_{1}\right)^{3}-\frac{r}{24} \alpha\left(M-\mu_{1}\right)^{3} \\
& \times\left(3 \mu_{1}+M\right)+\mathcal{O}\left(r^{2}\right),
\end{aligned}
$$

where $\mu_{1}$ is the scale parameterizing the division of the potential into the singular and regular parts. To obtain the singular part, we expanded the LO potential in a Taylor series of the mass $M$ around $M=\mu_{1}$ and kept the first three terms in the power series of $M-\mu_{1}$. Notice that since our toy model is not generated by a chirally invariant Lagrangian, we obtain a power series expansion in $M$ and not in $M^{2}$. As specified in Sect. 3, the number of subtracted terms when defining the regular potential $V_{R}$ is dictated by the condition that all diagrams generated by iterating Eq. (18) remain finite. We fix the renormalized coupling $c_{R}$ by the requirement to reproduce the low-energy phase shift of the underlying toy-model potential at a fixed value of the on-shell momentum $k$. Subtractions of loop integrals are performed at $E=-\mu^{2} /(1 \mathrm{GeV})$.

Our results for the phase shift for various choices of the renormalization scales are shown in Fig. 1 together with the phase shifts of the underlying toy-model to be regarded as synthetic data. The scale $\mu_{1}$ that parametrizes the ambiguity in the definition of the regular part of the potential should be chosen of the order of the hard scale in the problem. Using too soft values of the scale $\mu_{1}$ is, in general, expected to reduce the applicability range of the EFT. As seen from Fig. 1, one can find a range of values of the subtraction scales $\mu$ and $\mu_{1}$, for which the description of the synthetic data is reasonably good for the LO approximation. However, the results for the momenta $k \gtrsim M$ are sensitive to the changes of these values. As mentioned in Sect. 2, approximate perturbative expressions in QFTs always depend on the choice of the renormalization scheme.

To demonstrate that the observed reasonable description of the synthetic data within the employed subtractive renormalization scheme is not just an artifact of the specific choice of the coupling constant $\alpha$ in the underlying toy model, we also consider the cases corresponding to different values of $\alpha$. Specifically, by changing the sign of the coupling to $\alpha=36$, we arrive at a toy model with the attractive longrange potential. We also increase the strength of the longrange potential by a factor of two and consider the cases of $\alpha= \pm 72$. Our results for these three additional models are visualized in Fig. 2 for a number of representative choices of the scales $\mu$ and $\mu_{1}$. For a strong repulsive long-range interaction corresponding to $\alpha=-72$, we only show the results for $\mu_{1}=600 \mathrm{MeV}$ since the observed pattern is analogous to the already considered toy-model with $\alpha=-36$. Phase shifts corresponding to the attractive long-range potential are 

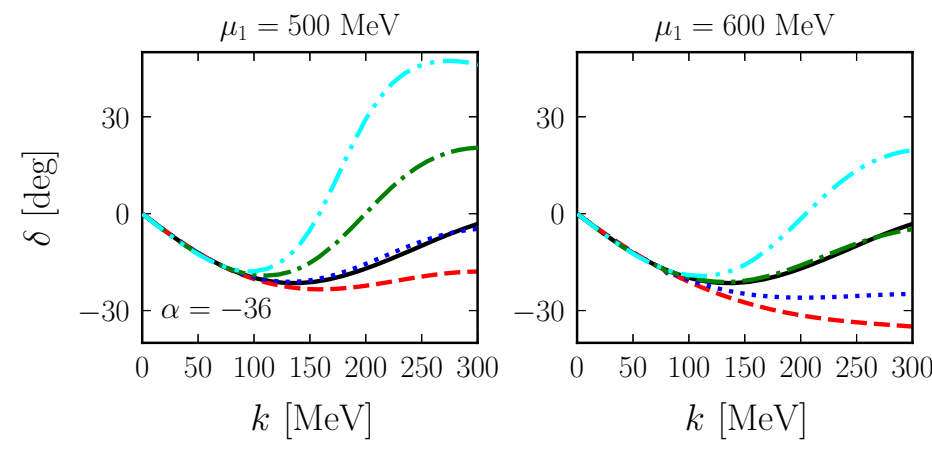

Fig. 1 The leading-order S-wave phase shift as a function of the centerof-mass momentum $k$. Solid (black) lines correspond to the underlying toy model while dashed lines show the LO phase shifts for various val-
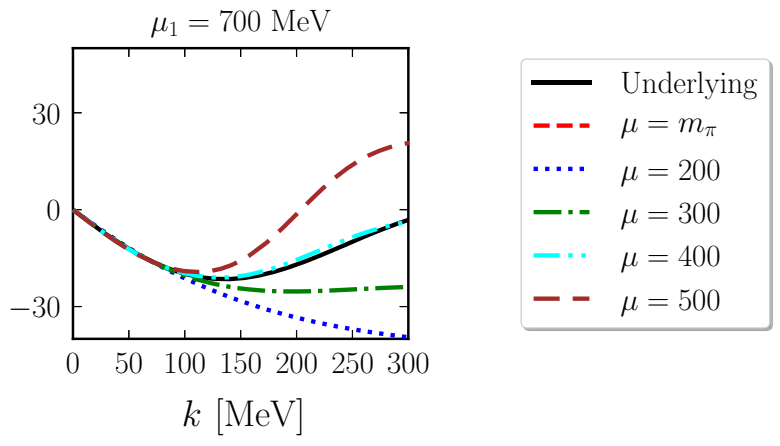

ues of $\mu$ and $\mu_{1}$. For each choice of the scale $\mu_{1}$, four representative choices for the scale $\mu$ are shown to demonstrate the trends

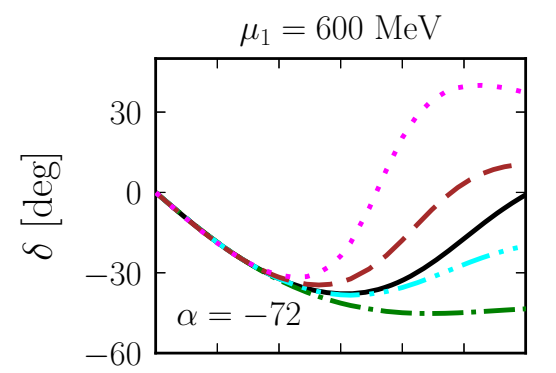

$$
\begin{aligned}
& \text { —Underlying - } \mu=400 \\
& -\boldsymbol{- \mu}=m_{\pi} \quad--\mu=500 \\
& \cdots \mu=200 \quad \cdots \mu=600 \\
& -* \mu=300 \quad-* \mu=700
\end{aligned}
$$
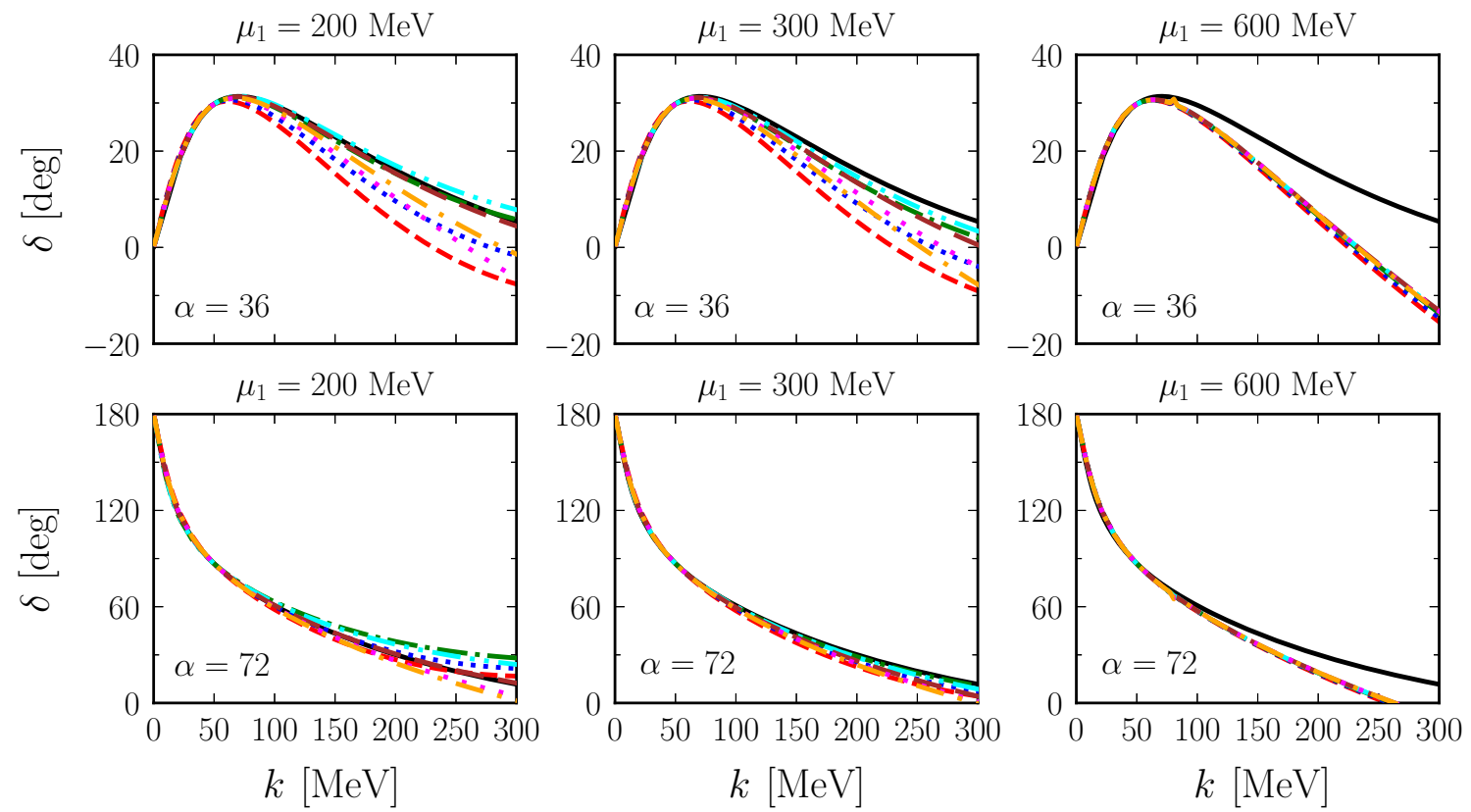

Fig. 2 The leading-order S-wave phase shifts as functions of the center-of-mass momentum $k$ for different values of the coupling $\alpha$. Solid (black) lines correspond to the underlying toy model while dashed lines show the LO phase shifts for various values of $\mu$ and $\mu_{1}$

plotted in the second and third rows of Fig. 2. The behavior of the phase shifts indicates the appearance of a low-lying virtual (real) bound state for $\alpha=36(\alpha=72)$. Compared to the repulsive case, we find an even larger range of subtraction scales leading to a reasonable description of the phase shifts. In these particular cases, softening the scale $\mu_{1}$ effectively amounts to a resummation of the subleading contact interaction which helps to improve the reproduction of the effective range. ${ }^{3}$ Still, choosing $\mu_{1}$ of the order of the hard scale such as e.g. $\mu_{1}=600 \mathrm{MeV}$ leads to an adequate LO description of the synthetic data. Furthermore, fully in line with the findings of Ref. [31], the scale $\mu$ can be varied in

\footnotetext{
${ }_{3}$ Notice that in the realistic case of neutron-proton scattering in the ${ }^{1} \mathrm{~S}_{0}$ channel, the subleading contact interaction was argued to require a nonperturbative treatment in Ref. [31].
} 
a broad range and set to soft values since the systems under consideration are not too close to the unitary limit.

\section{Summary}

In this paper we discussed the problem of renormalization of singular potentials in the low-energy effective field theory of the strong interactions. We outlined the standard procedure of quantum field theoretical renormalization emphasizing its general definition that applies to both non-perturbative as well as perturbative calculations. The main message of our work is that chiral EFT in the few-nucleon sector does not require the invention of any new non-perturbative renormalization that would go beyond what is already well-established in quantum field theory.

To demonstrate that the problem lies not in conceptual issues but rather in the complicated technical implementation of the standard renormalization approach, we proposed a way of implementing the subtractive renormalization for solutions of integral equations with singular potentials. The suggested renormalization scheme corresponds to subtracting all divergences appearing at a given order, and also (over)subtracting finite pieces with some fixed normalization conditions. While being just one of infinitely many possibilities of fixing the normalization conditions, and very likely not the best one, the suggested scheme has the advantage of being easily applicable in practice and serves well our purposes of demonstrating a self-consistent EFT renormalization.

As an example to demonstrate these rather general statements, we applied the considered subtractive renormalization scheme to the toy model of Ref. [12]. While there exists a region of values of the renormalization scales for which the description of the "data" is good for the LO approximation, the results are sensitive to the changes of these values as one expects on general grounds.

Acknowledgements Open Access funding provided by Projekt DEAL. This work was supported in part by BMBF (Grant No. 05P18PCFP1), by DFG (Grant No. 426661267), by DFG and NSFC through funds provided to the Sino-German CRC 110 "Symmetries and the Emergence of Structure in QCD" (NSFC Grant No. 11621131001, DFG Grant No. TRR110), by the Georgian Shota Rustaveli National Science Foundation (Grant No. FR17-354), by VolkswagenStiftung (Grant no. 93562) and by the CAS President's International Fellowship Initiative (PIFI) (Grant No. 2018DM0034).

Data Availability Statement This manuscript has no associated data or the data will not be deposited. [Authors' comment: All the data are contained in the figures.]

Open Access This article is licensed under a Creative Commons Attribution 4.0 International License, which permits use, sharing, adaptation, distribution and reproduction in any medium or format, as long as you give appropriate credit to the original author(s) and the source, provide a link to the Creative Commons licence, and indicate if changes were made. The images or other third party material in this article are included in the article's Creative Commons licence, unless indi- cated otherwise in a credit line to the material. If material is not included in the article's Creative Commons licence and your intended use is not permitted by statutory regulation or exceeds the permitted use, you will need to obtain permission directly from the copyright holder. To view a copy of this licence, visit http://creativecomm ons.org/licenses/by/4.0/.

\section{References}

1. S. Weinberg, Phys. Lett. B 251, 288 (1990)

2. P.F. Bedaque, U. van Kolck, Ann. Rev. Nucl. Part. Sci. 52, 339 (2002). arXiv:nucl-th/0203055

3. E. Epelbaum, Prog. Part. Nucl. Phys. 57, 654 (2006). arXiv:nucl-th/0509032

4. E. Epelbaum, H.-W. Hammer, U.-G. Meißner, Rev. Mod. Phys. 81, 1773 (2009). arXiv:0811.1338 [nucl-th]

5. M.C. Birse, PoS CD 09, 078 (2009). arXiv:0909.4641 [nucl-th]

6. R. Machleidt, D.R. Entem, Phys. Rept. 503, 1 (2011). arXiv:1105.2919 [nucl-th]

7. E. Epelbaum, U.-G. Meißner, Ann. Rev. Nucl. Part. Sci. 62, 159 (2012). arXiv:1201.2136 [nucl-th]

8. M.P. Valderrama, Int. J. Mod. Phys. E 25, 1641007 (2016). arXiv: 1604.01332 [nucl-th]

9. H.-W. Hammer, S. König and U. van Kolck, arXiv:1906.12122 [nucl-th]

10. R. Machleidt, F. Sammarruca, arXiv:2001.05615 [nucl-th]

11. S. Weinberg, "The Quantum Theory Of Fields. Vol. 1,2: Foundations, Modern applications" Cambridge, UK: Univ. Pr. (1995)

12. E. Epelbaum, A.M. Gasparyan, J. Gegelia, U.-G. Meißner, Eur. Phys. J. A 54, 186 (2018). arXiv:1810.02646 [nucl-th]

13. G. P. Lepage, arXiv:nucl-th/9706029

14. U. van Kolck, arXiv:2003.06721 [nucl-th]

15. S.R. Beane, T.D. Cohen, D.R. Phillips, Nucl. Phys. A 632, 445 (1998). arXiv:nucl-th/9709062

16. G. P. Lepage, Conference summary, Prepared for INT Workshop on Nuclear Physics with EFT, Seattle, Washington, 25-26 Feb 1999

17. J. Gegelia, J. Phys. G 25, 1681 (1999). arXiv:nucl-th/9805008

18. J. Gegelia, S. Scherer, Int. J. Mod. Phys. A 21, 1079 (2006). arXiv:nucl-th/0403052

19. E. Epelbaum, U.-G. Meißner, Few Body Syst. 54, 2175 (2013). arXiv:nucl-th/0609037

20. E. Epelbaum, H. Krebs and P. Reinert, arXiv:1911.11875 [nucl-th]

21. J. C. Collins, "Renormalization. An introduction to renormalization, the renormalization group, and the operator product expansion," Cambridge, Uk: Univ. Pr. (1984) 380p

22. J. Gegelia, Phys. Lett. B 429, 227 (1998)

23. T. Frederico, V.S. Timoteo, L. Tomio, Nucl. Phys. A 653, 209 (1999). arXiv:nucl-th/9902052

24. V.S. Timoteo, T. Frederico, A. Delfino, L. Tomio, Phys. Lett. B 621, 109 (2005). arXiv:nucl-th/0508006

25. V.S. Timoteo, T. Frederico, A. Delfino, L. Tomio, Phys. Rev. C 83, 064005 (2011). arXiv:1006.1942 [nucl-th]

26. C.-J. Yang, C. Elster, D.R. Phillips, Phys. Rev. C 77, 014002 (2008). arXiv:0706.1242 [nucl-th]

27. C.J. Yang, C. Elster, D.R. Phillips, Phys. Rev. C 80, 034002 (2009) arXiv:0901.2663 [nucl-th]

28. W. Frank, D.J. Land, R.M. Spector, Rev. Mod. Phys. 43, 36 (1971). https://doi.org/10.1103/RevModPhys.43.36

29. J. Gegelia, Phys. Lett. B 463, 133 (1999). arXiv:nucl-th/9908055

30. E. Epelbaum, J. Gegelia, U.-G. Meißner, Nucl. Phys. B 925, 161 (2017). arXiv:1705.02524 [nucl-th]

31. E. Epelbaum, A.M. Gasparyan, J. Gegelia, H. Krebs, Eur. Phys. J. A 51, 71 (2015). arXiv:1501.01191 [nucl-th] 